\title{
Parámetros de corte en brocas genéricas para su aplicación en pequeñas y medianas empresas metalmecánicas del Ecuador
}

\author{
Victor A. Erazo-Arteaga, Marjorie P. Segovia, Jonathan X. Lima, Cosme D. Mejía-Echeverría \\ (1) Facultad de Ingeniería en Ciencias Aplicadas, Carrera de Ingeniería Mecatrónica, Universidad Técnica del Norte, Av. \\ 17 de Julio 5-21, Ibarra-Ecuador (correo-e: vaerazo@utn.edu.ec; mpsegovias@utn.edu.ec; jxlima@utn.edu.ec; \\ cdmejia@utn.edu.ec)
}

Recibido May. 24, 2021; Aceptado Jul. 21, 2021; Versión final Ago. 15, 2021, Publicado Dic. 2021

\begin{abstract}
Resumen
El presente estudio tiene como objetivo optimizar la vida útil de herramientas genéricas de taladrado que se utilizan de forma empírica en micro, pequeñas y medianas empresas (MIPYME) metalmecánicas del Ecuador. Primero, se miden la preferencia de las herramientas genéricas, se adquieren las brocas de mayor aceptación y se propone un conjunto de parámetros de corte. Posteriormente, se realizan ensayos en los que se observa el desgaste de flanco y la potencia de corte consumida. Finalmente, se repite el ensayo para condiciones de corte generalmente utilizadas por los operadores. Los resultados muestran que las brocas genéricas presentan una composición semejante al acero de herramientas JIS-SHK55. Se concluye que con los parámetros de corte propuestos, la vida útil de las herramientas se alarga, pero presentan un mayor consumo de potencia.
\end{abstract}

Palabras clave: taladrado; MIPYME; velocidad; corte; desgaste; herramienta; potencia

\section{Cutting parameters in generic drills for applications in small and medium-sized metalworking companies from Ecuador}

\begin{abstract}
The primary objective of this study is to optimize the lifetime of generic drilling tools used in micro, small, and medium-sized (MIPYME, in Spanish) metalworking companies in Ecuador. First, the preference of generic tools is assessed, the most widely accepted drills are acquired, and a set of cutting parameters is proposed. Then, tests are performed to measure flank wear and consumed power. Finally, tests are repeated to assess cutting conditions used by operators. The results show that generic drills have a composition similar to that of the JIS-SHK55 tool steel. It is concluded that by using the cutting parameters proposed here, drilling tool lifetime is increased, but with greater power consumption.
\end{abstract}




\section{INTRODUCCIÓN}

En el Ecuador existen más de 8000 empresas metalmecánicas, de las cuales el 99\% pertenecen a las micro, pequeñas y medianas empresas (MIPYMES), este sector brinda empleo a más de 21000 personas jugando así un rol importante dentro de la economía interna del país. Por lo general, estas microempresas carecen de conocimientos técnicos y su funcionamiento se basa en la experiencia, los principales inconvenientes del sector radican en la falta de una estructura organizativa y deficiente gestión en sus procesos de manufactura (Quezada-Torres et al., 2017; Quezada-Torres et al., 2019). Por otra parte, China se ha convertido en socio comercial estratégico para América latina, debido a su alta capacidad productiva, desde este país es posible importar a bajo costo varios bienes que permiten la creación de un taller metalmecánico. A nivel mundial, China ocupa el $16 \%$ de las exportaciones de productos de acero, según informa Veritradecorp, el $44 \%$ de las herramientas de corte para taladrado provienen de este país. $Y$ de todas las brocas que ingresan al Ecuador, el 34\% no poseen marca comercial alguna (Arteaga et al., 2020; Liu et al., 2019).

Se plantea como hipótesis; que las herramientas de taladrado genéricas que se comercializan en el mercado nacional serán utilizadas en MIPYMES metalmecánicas. Y que su utilización se realiza en base a la experiencia laboral del operador, ya que se desconoce el material del cual están fabricadas. Si se utilizan parámetros de corte equivocados la herramienta colapsa prematuramente (Lobos \& Guzmán, 2019; Nickel et al., 2000), entonces, se puede incurrir en gastos adicionales debido a los tiempos dedicados a la recuperación del filo; y al final, la herramienta será reemplazada por otra de iguales características. Surge entonces la necesidad de plantear estrategias que permitan incrementar la productividad y fomentar un desarrollo sostenible, a través de la adquisición de nuevos conocimientos teóricos y la puesta en práctica de estos (Tseng et al., 2019). Con la finalidad de optimizar los procesos de taladrado se han presentado varias investigaciones. Se tiene que en el año 2006 se realiza un estudio de confiabilidad para herramientas de taladrado donde el objetivo es encontrar la distribución de probabilidad para el desgaste de una herramienta de acero AISI M2. Sin fluido de corte, utilizando los parámetros recomendados por el fabricante de la herramienta y con relación longitud-diámetro (l/d) igual a 5 , se realizaron agujeros de $5 \mathrm{~mm}$ de diámetro sobre una probeta de acero AISI 1016. Tomando como referencia un desgaste de flanco (VB) máximo de $0.3 \mathrm{~mm}$ se alcanzó una longitud de corte de 1125 mm (Patiño \& Martha de Souza, 2006).

Garg et al., (2010) plantean la predicción del desgaste en herramientas de taladrado utilizando inteligencia artificial, experimentando con brocas de acero de alta velocidad HSS, una probeta de acero de bajo carbono y una profundidad de agujero de $15 \mathrm{~mm}$. En los ensayos realizados, una herramienta diámetro $8 \mathrm{~mm}$ alcanza una longitud de corte de $390 \mathrm{~mm}$, trabajando con $360 \mathrm{rpm}$ y avance de $0.25 \mathrm{~mm} / \mathrm{rev}$. Una herramienta diámetro $10 \mathrm{~mm}$ alcanza $135 \mathrm{~mm}$ de longitud, trabajando con $900 \mathrm{rpm}$ y avance de $0.25 \mathrm{~mm} / \mathrm{rev}$. El desgaste VB de la primera herramienta fue semejante a $0.26 \mathrm{~mm}$ y de la segunda $0.37 \mathrm{~mm}$. Neugebauer et al., (2011) en su artículo investigan la forma de aumentar la eficiencia energética en los procesos de manufactura por arranque de viruta, en base a la correcta selección de los parámetros de corte. Sobre probetas de fundición EN-GJL250 se realizaron agujeros de $7 \mathrm{~mm}$ de diámetro con profundidad $35 \mathrm{~mm}$, utilizando una broca de acero HSSE. La potencia de la máquina es prácticamente constante (aproximadamente $700 \mathrm{~W}$ ) y la vida útil de la herramienta alcanza $3780 \mathrm{~mm}$. Se estipula que el taladrado en seco con altos caudales de corte es inadecuado para la optimización de energía y recursos.

Marques et al., (2015) comparan la vida útil entre brocas de acero AISI M2 sin recubrimiento, y con recubrimiento de Nitruro de Aluminio Cromo (AICrN). Utilizando los parámetros de corte recomendados por los fabricantes de las brocas, se realizan taladrados de 8 milímetros sobre placas de fundición nodular DIN GGG 50. Se obtienen 128 agujeros de $48 \mathrm{~mm}$ de profundidad, las brocas recubiertas alcanzaron un desgaste VB de $0.26 \mathrm{~mm}$, mientras que en las herramientas sin recubrimiento fue de $0.36 \mathrm{~mm}$. Puneet et al., (2017) estudian la influencia de la superficie en la vida útil de las brocas con recubrimiento de nitruro de titanio, se determinó que las brocas de HSS sin revestimiento tienen una vida útil de $1575 \mathrm{~mm}$ en probetas de acero EN-24 con VB de $0.35 \mathrm{~mm}$. Se utilizó una broca de $6 \mathrm{~mm}$ de diámetro, con una velocidad de corte de 30 $\mathrm{m} / \mathrm{min}$ y un avance de $0.1 \mathrm{~mm} / \mathrm{rev}$. Además, se demostró que con su propuesta la vida de la herramienta es el triple en comparación con las herramientas disponibles en el mercado.

Khan et al., (2020) en su investigación utilizaron aceite de corte al experimentar con brocas diámetro $5 \mathrm{~mm}$ de aceros M2, M42 y M35 sobre probetas de AISI 304. En los tres casos se utilizó una profundidad de corte de $20 \mathrm{~mm}$ y avance de $0.1 \mathrm{~mm} / \mathrm{rev}$. En cuanto a la vida útil, en la broca de acero M2 se alcanzó $960 \mathrm{~mm}$ con velocidad de corte $11 \mathrm{~m} / \mathrm{min}$. En la herramienta de acero M42 la vida fue de $480 \mathrm{~mm}$ utilizando $9 \mathrm{~m} / \mathrm{min}$. En la herramienta de acero M35 se utilizó $3 \mathrm{~m} / \mathrm{min}$ y la longitud de corte alcanzada fue $60 \mathrm{~mm}$. Las perforaciones se realizaron hasta que la herramienta de corte presente un desgaste de flanco de $0.2 \mathrm{~mm}$ en todos los casos.Pena et al., (2005) realizan un monitoreo y evaluación de la formación de rebabas internas no deseadas durante operaciones de perforación, basados en un grupo de señales que se transmiten producto del torque empleado por el husillo para esta operación. Para esto utilizaron en los experimentos Aluminio Al 7075-T6 sin lubricación y a altas velocidades Se detectó una influencia directa entre la altura de la rebaba y la 
intensidad y características de la señal monitoreada, en contraposición a los cambios de parámetros del proceso, los que no tuvieron una gran influencia en dichas señales. Los autores proponen este método de monitoreo como control de calidad en las operaciones de perforación de metales.

Olvera et al., (2012) describen estudios relacionados con el fresado de bolas helicoidales para realizar orificios en una pieza de metal de aleación de Titanio Ti-6Al-4V utilizando para esto dos estrategias de mecanizado diferentes: una con una trayectoria de fresado para la obtención del diámetro nominal y la segunda basada en dos etapas, primera etapa con diámetro de 50 micras por debajo del nominal y la segunda la eliminación del resto del material utilizando el flanco de la herramienta. Se evaluaron parámetros tales como tiempo de proceso, calidad de acabado del agujero, diámetro, rugosidad y formación de rebabas a la entrada y salida del orificio. Estos estudios arrojaron como resultados que se producen rebabas insignificantes, siendo versátiles para estas operaciones. No obstante observaron que la dureza en las zonas cercanas a las superficies internas mecanizadas con herramientas de fresas de bolas, disminuye con respecto al mecanizado de perforación mediante torsión.

Lacalle et al., (2011) plantean en su artículo un caso de estudio basado en la fabricación de componentes de alta resistencia y funcionabilidad con la utilización de brocas helicoidales de longitud considerable (Depp Twist Drilling). Esta técnica de perforación permite la construcción de componentes estructurales con una alta reducción de masa y reducción de sus tensiones internas comparadas con piezas soldadas o atornilladas. A través de un proceso de monitorización de la operación de taladrado los autores optimizaron el mismo y por ende se permitió una mejora de las condiciones de corte y rediseño de los componentes estructurales a los que se les aplica esta novedosa técnica, lográndose además un aumento de la confiabilidad en el uso sistemático de este proceso en la industria. Los estudios se establecieron bajo el análisis de los Métodos de Elementos Finitos (FEM) y los resultados mostraron alta resistencia a las fuerzas cortantes y de compresión de los elementos estructurales estudiados.

En el caso de la mayoría de la bibliografía publicada se conoce el tipo de herramientas utilizadas y las condiciones de corte con las que se trabajó, o las mismas son recomendadas por los fabricantes de las herramientas, a diferencia de las herramientas de taladrado genéricas que no cuentan con esa información que permita una optimización del proceso de manufactura. Por esta razón se propone caracterizar las herramientas genéricas de taladrado y experimentar con ellas para determinar los parámetros de corte adecuados y optimizar su vida útil.

\section{METODOLOGÍA}

Con la intención de evaluar la aceptación de las herramientas genéricas, se recopila información mediante la encuesta mostrada en la Tabla 1. Para conocer el tipo de broca comúnmente utilizada y los parámetros de corte empíricos, se muestra la Fig. 1 y se da a escoger un rango de velocidades que se han tomado de las publicaciones de Cselle \& Barimani, (1995) y Amini et al., (2017).

Tabla 1. Encuesta sobre el uso de brocas genéricas.

\begin{tabular}{|c|c|}
\hline Pregunta & Opción \\
\hline \multirow[t]{3}{*}{ En su organización se compran brocas: } & De marca \\
\hline & Genéricas (sin marca) \\
\hline & Las dos \\
\hline \multirow[t]{2}{*}{ De estas brocas genéricas, usted ¿Cuál prefiere? } & Opción 1 \\
\hline & Opción 2 \\
\hline \multirow{2}{*}{$\begin{array}{l}\text { Para los parámetros de corte, ¿prefiere usar su } \\
\text { experiencia en lugar de libros o catálogos? }\end{array}$} & $\mathrm{Si}$ \\
\hline & No \\
\hline \multirow[t]{3}{*}{ En su organización, los diámetros de brocas son: } & hasta $12 \mathrm{~mm}$ \\
\hline & hasta $25 \mathrm{~mm}$ \\
\hline & mayores a $25 \mathrm{~mm}$ \\
\hline \multirow{4}{*}{$\begin{array}{l}\text { Para taladrar con la broca diámetro } 8 \mathrm{~mm} \text { a usted } \\
\text { mostrada, ¿en qué rango esta la velocidad de husillo? }\end{array}$} & entre 400 a $500 \mathrm{rpm}$ \\
\hline & entre 700 a $1200 \mathrm{rpm}$ \\
\hline & entre 1200 a $1900 \mathrm{rpm}$ \\
\hline & superior a $1900 \mathrm{rpm}$ \\
\hline
\end{tabular}

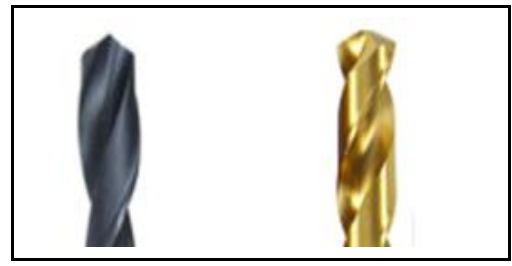

Fig. 1: Imagen de encuesta. A la izquierda opción 1, a la derecha opción 2. 
El estudio presentado por Quezada-Torres et al., (2017) revela que en la ciudad de Ibarra existen 69 microempresas metalmecánicas, se plantea realizar el levantamiento de información en al menos 58 empresas, que representa una confiabilidad del $95 \%$. Las restricciones emitidas por la pandemia declarada a nivel mundial impidieron obtener la muestra total, se logra recopilar información de 45 empresas que representan el $92 \%$ de confiabilidad. Sabiendo que Quito es una de las ciudades donde se ubican los principales distribuidores del Ecuador (Urdaneta \& Borgucci, 2021), diferentes almacenes son seleccionados al azar para adquirir brocas de diámetro 5, 8, 10 y $12 \mathrm{~mm}$. Para caracterizar estas herramientas, sobre las brocas diámetro $12 \mathrm{~mm}$ se mide la dureza y su composición química, utilizando un durómetro HBRV-187.5 y un espectrómetro Q2 ION. Para estimar los parámetros de corte a utilizar en los ensayos, se toman en cuenta las ecuaciones (Vazquez et al., 2018) :

$$
\begin{aligned}
& V c=\pi \cdot D \cdot N \\
& N=\frac{L c}{T c \cdot a} \\
& a=\frac{2 \cdot S c}{D}
\end{aligned}
$$

En los modelos de fuerza de corte expuestos por (Polini \& Prisco, 2003), la sección de viruta es de 1 mm², al relacionar este dato con las tres ecuaciones anteriores se tiene:

$$
\mathrm{Vc}=\frac{\pi \cdot D^{2} \cdot \mathrm{Lc}}{2 \cdot \mathrm{TC}}
$$

Según los ensayos realizados por Marques et al., (2015), una broca recubierta puede alcanzar una longitud de corte máxima de $6144 \mathrm{~mm}$, trabajando sin fluido de corte. Para el tiempo de corte se estima una hora, sugerido por la ecuación de Taylor, entonces, reemplazando estos datos se calculan los parámetros de corte que se muestran en la Tabla 2. Para contrastar esta propuesta, se utilizan los parámetros de corte mostrados en la Tabla 3, mismos que fueron estimados a partir de la encuesta realizada a los metalmecánicos que utilizan parámetros de corte en base a su experiencia.

Tabla 2. Parámetros de corte propuestos.

\begin{tabular}{|c|c|c|}
\hline $\begin{array}{c}\text { Diámetro de broca } \\
\mathrm{mm}\end{array}$ & $\begin{array}{c}\text { Velocidad de corte } \\
\mathrm{m} / \mathrm{min}\end{array}$ & $\begin{array}{c}\text { Avance } \\
\mathrm{mm} / \mathrm{rev}\end{array}$ \\
\hline 5 & 4.021 & 0.4 \\
\hline 8 & 10.29 & 0.25 \\
\hline 10 & 16.08 & 0.2 \\
\hline
\end{tabular}

Tabla 3. Parámetros de corte empíricos

\begin{tabular}{|c|c|c|}
\hline $\begin{array}{c}\text { Diámetro de broca } \\
\mathrm{mm}\end{array}$ & $\begin{array}{c}\text { Velocidad de corte } \\
\mathrm{m} / \mathrm{min}\end{array}$ & $\begin{array}{c}\text { Avance } \\
\mathrm{mm} / \mathrm{rev}\end{array}$ \\
\hline 5 & 8 & 0.07 \\
\hline 8 & 13.2 & 0.106 \\
\hline 10 & 14 & 0.13 \\
\hline
\end{tabular}

Como probetas, se utilizan placas de acero ASTM A36 a las cuales se realiza un planeado de $2 \mathrm{~mm}$ de profundidad, con la finalidad de medir dureza en varios puntos, como se muestra la Fig. 2. Las medidas de las probetas y el número de mediciones se indica en la Tabla 4. Para ejecutar el ensayo de taladrado se utiliza un centro de mecanizado CNC Romi D800, con software CAD/CAM se planifica la posición de los agujeros, se marca cada centro con una broca DIN 333 de $8 \mathrm{~mm}$ de diámetro y se realiza el taladrado respetando los parámetros mencionados, sin desahogo de viruta y sin fluido de corte. La potencia consumida se registra utilizando la tabla de consumo energético de la máquina CNC.

Debido a la naturaleza de los trabajos realizados por mencionadas organizaciones metalmecánicas, para las brocas de diámetro 5 se utiliza una relación de taladrado no profundo (l/d) igual a 2, para las brocas 8 y 10 se utiliza una relación de 2.5 (Biermann et al., 2012). Para este estudio se considera VB superior a $0.4 \mathrm{~mm}$ como el criterio de vida útil de la herramienta (Garg et al., 2010). Se mide el desgaste cada 10 agujeros utilizando un microscopio digital que es acoplado a la máquina con un soporte impreso en 3D según el ángulo en la punta de las brocas. Tal como se indica en la Fig. 3, para obtener esta característica se comparó las herramientas adquiridas con galgas estandarizadas. 


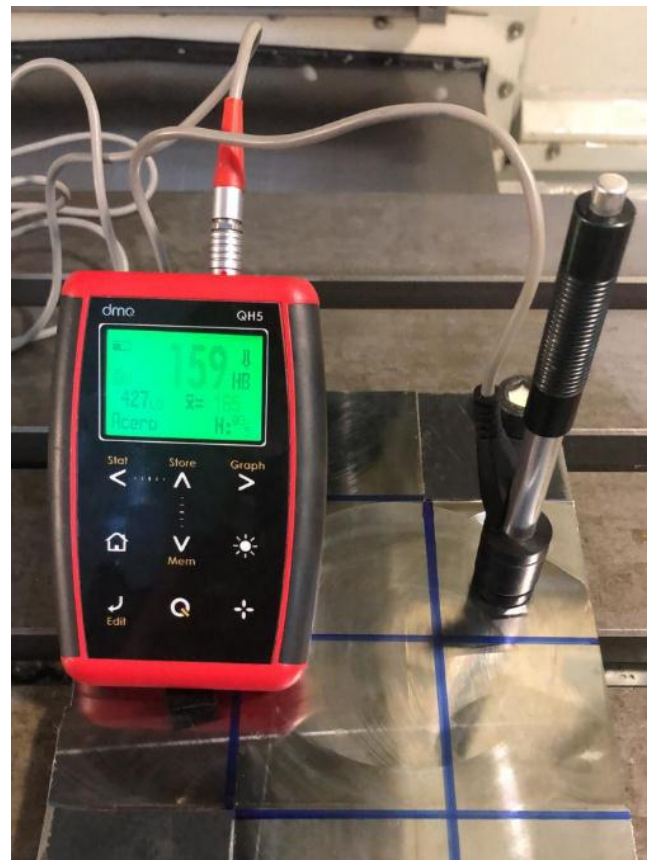

Fig. 2: Medición de dureza en placas de pruebas.

Tabla 4. Medición de dureza en placa.

\begin{tabular}{|c|c|c|c|}
\hline $\begin{array}{c}\text { Diámetro de broca } \\
m m\end{array}$ & $\begin{array}{c}\text { Tamaño de probeta } \\
m m\end{array}$ & $\begin{array}{c}\text { Numero de muestras } \\
\text { tomadas }\end{array}$ & $\begin{array}{c}\text { Cantidad de } \\
\text { probetas }\end{array}$ \\
\hline 5 & $210 \times 160 \times 11$ & 10 & 3 \\
\hline 8 & $276 \times 182 \times 21$ & 12 & 2 \\
\hline 10 & $298 \times 180 \times 25$ & 20 & 3 \\
\hline
\end{tabular}
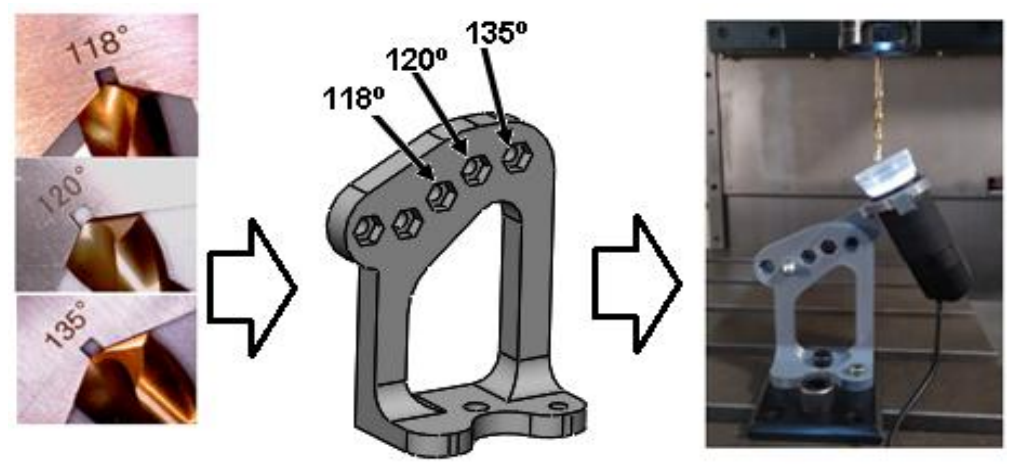

Fig. 3: Adaptación de microscopio en función del ángulo de herramienta.

\section{RESULTADOS Y DISCUSIÓN}

Los datos obtenidos en la encuesta se muestran en la Fig. 4, se puede observar que el $55.6 \%$ utiliza los dos tipos de brocas y el $28.9 \%$ utiliza solo brocas genéricas. El $71.2 \%$ reconoce utilizar las herramientas de forma empírica. Se observa que el $84.5 \%$ prefiere utilizar brocas recubiertas (opción 2, Fig.1) y que el $77 \%$ de encuestados seleccionó un rango de 400 a 500 rpm como velocidad angular adecuada para una broca diámetro $8 \mathrm{~mm}$.

La gran cantidad de materiales que existente, dificulta saber con exactitud la norma a la que se rige el material de la broca ensayada. Por ser la más completa, se utiliza la base de datos MatWeb para comparar los resultados de la espectrometría con otros aceros de herramientas (Ramalhete et al., 2010), no se pudo determinar el material y espesor del recubrimiento debido a las características del ensayo. Como se muestra en la Tabla 5, la composición resultante es similar a los aceros de herramientas JIS SHK55, AISI M36 y AISI M41. El análisis de dureza arrojó como resultado $705 \mathrm{HB}$. Una de las características de las herramientas de taladrado es el ángulo de la punta, este depende del material a trabajar y por lo general es el fabricante quien lo recomienda (Berzosa et al., 2020). 
Velocidad de husillo entre 400 a $500 \mathrm{r} / \mathrm{min}$ Velocidad de husillo entre 700 a $1200 \mathrm{r} / \mathrm{min}$ Velocidad de husillo entre 1200 a $1900 \mathrm{r} / \mathrm{min}$

Utilizan brocas de hasta $25 \mathrm{~mm}$ Utilizan brocas de hasta $12 \mathrm{~mm}$ Utilizan brocas mayores a $25 \mathrm{~mm}$

Parámetros de corte en base a experiencia Parámetros de corte publicados o recomendados

Uso de brocas con recubrimiento Uso de brocas sin recubrimiento

Uso de brocas de marcas y genéricas Uso de brocas genéricas Uso de brocas de marca

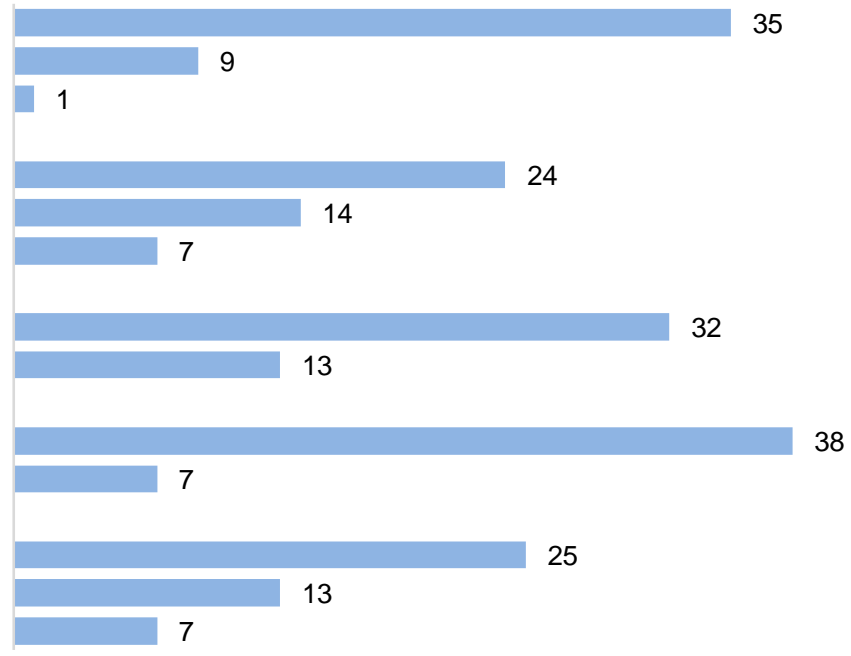

38

Fig. 4: Resultados de la encuesta.

Tabla 5. Composición de las brocas genéricas.

\begin{tabular}{|l|c|c|c|c|c|c|c|c|c|}
\hline \multirow{2}{*}{ Material } & \multicolumn{9}{c|}{ Composición química \% } \\
\cline { 2 - 11 } & $C$ & $M n$ & $P$ & $S$ & $S i$ & $C r$ & $W$ & Mo & $V$ \\
\hline Ensayo & 0.914 & 0.305 & 0.003 & 0.0055 & 0.123 & 3.603 & 10.51 & 5.768 & 1.452 \\
\hline AISI M36 & $0.8-0.9$ & $0.15-0.40$ & $<=0.030$ & $<=0.030$ & $0.20-0.45$ & $3.75-4.0$ & $5.5-6.5$ & $4.5-5.5$ & $1.75-2.25$ \\
\hline AISI M41 & $1.05-1.15$ & $0.20-0.60$ & $<=0.030$ & $<=0.030$ & $0.15-0.50$ & $3.75-4.0$ & $6.25-7.0$ & $3.25-4.25$ & $1.75-2.25$ \\
\hline JIS SHK55 & $0.85-0.95$ & $<=0.40$ & $<=0.030$ & $<=0.030$ & $<=0.40$ & $4.5-5.5$ & $5.7-6.7$ & $4.6-5.3$ & $1.7-2.3$ \\
\hline
\end{tabular}

Se pudo detectar que el ángulo en la punta de las brocas genéricas oscila entre $118^{\circ}$ y $135^{\circ}$, tal como se indicó en la Fig. 3, para los ensayos se utilizaron solamente aquellas que calzaban perfectamente en las galgas, la Tabla 6 muestra la cantidad de brocas que poseen ángulos exactos y ángulos intermedios. Para caracterizar estas herramientas, se podría decir que; son brocas helicoidales fabricadas de acero JIS SHK55, con una dureza 705 HB y un ángulo de punta de $120^{\circ}$. Sobre las probetas de acero ASTM A36 se realizaron 114 mediciones de dureza, los resultados se resumen en la Tabla 7. Al mirar la Fig. 5 se puede constatar que la dureza de suministro estipulada por el distribuidor es inferior a las obtenidas en el ensayo. En condiciones normales de trabajo, esto afecta la selección de parámetros de corte y por consiguiente la vida útil de las herramientas. Para las brocas diámetro $5 \mathrm{~mm}$, con los parámetros propuestos se obtuvo una longitud de corte de $2200 \mathrm{~mm}$ y un tiempo de corte de $21.49 \mathrm{~min}$. Se registró una potencia mínima de $149 \mathrm{~W}$ y una máxima de $200 \mathrm{~W}$. Con los parámetros empíricos, se obtuvo una longitud de corte de $400 \mathrm{~mm}$ y un tiempo de corte de $11.22 \mathrm{~min}$. Se registró una potencia mínima de 137 W y una máxima de $172 \mathrm{~W}$.

Tabla 6. Numero de brocas al as que se midió el ángulo.

\begin{tabular}{|c|c|c|c|c|}
\hline Brocas $\mathrm{mm}$ & $118^{\circ}$ & $120^{\circ}$ & $135^{\circ}$ & Ángulos intermedios \\
\hline 5 & 1 & 3 & 0 & 1 \\
\hline 8 & 0 & 3 & 0 & 1 \\
\hline 10 & 0 & 3 & 1 & 0 \\
\hline 12 & 0 & 0 & 3 & 0 \\
\hline
\end{tabular}

Tabla 7. Resultado de dureza en las probetas de ASTM A36.

\begin{tabular}{|c|c|c|c|}
\hline \multirow{2}{*}{ Ensayo } & \multirow{2}{*}{ Probeta número } & \multicolumn{2}{|c|}{ Dureza (HB) } \\
\cline { 2 - 4 } & & Máxima & Mínima \\
\hline \multirow{3}{*}{ Broca $5 \mathrm{~mm}$} & 1 & 179 & 159 \\
\cline { 2 - 4 } & 2 & 196 & 159 \\
\cline { 2 - 4 } & 3 & 352 & 189 \\
\hline \multirow{2}{*}{ Broca $8 \mathrm{~mm}$} & 4 & 353 & 93 \\
\cline { 2 - 4 } & 5 & 324 & 102 \\
\hline \multirow{3}{*}{ Broca $10 \mathrm{~mm}$} & 6 & 162 & 136 \\
\cline { 2 - 4 } & 7 & 329 & 203 \\
\cline { 2 - 4 } & 8 & 334 & 209 \\
\hline
\end{tabular}




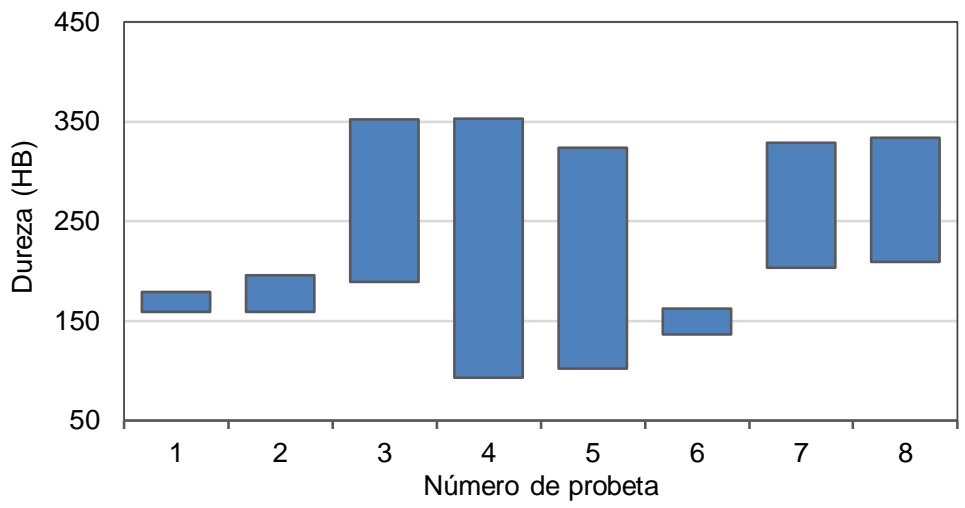

Fig. 5: Dureza en probetas de taladrado.

La variación de potencia con relación a la longitud de corte para los dos casos se muestra en la Fig. 6 . El desgaste con respecto a la longitud de corte se puede observar en la Fig. 7, la forma del desgaste y su posición se indican en la Fig. 8. Los parámetros de corte empíricos son deficientes en comparación con los recomendados por los fabricantes de otras herramientas, las publicaciones de Khan et al., (2020), Patiño \& Martha de Souza, (2006) así lo demuestran, en el primer caso, pese que la velocidad de corte es 8 veces mayor se triplica la vida útil de la herramienta. En el segundo caso, la vida útil con parámetros empíricos es $80 \%$ inferior. Se debe puntualizar que los autores mencionados utilizan aceros ligeramente más suaves. En cuanto a los parámetros propuestos, los resultados son satisfactorios debido a que se obtiene $6 \%$ más vida útil que la presentada por Khan et al., (2020) y $7 \%$ inferior a la vida útil publicada por Puneet et al., (2017) para un acero de igual dureza. Sin embargo, con relación a este último se propone una velocidad de avance $60 \%$ superior lo que implica menor tiempo de corte.

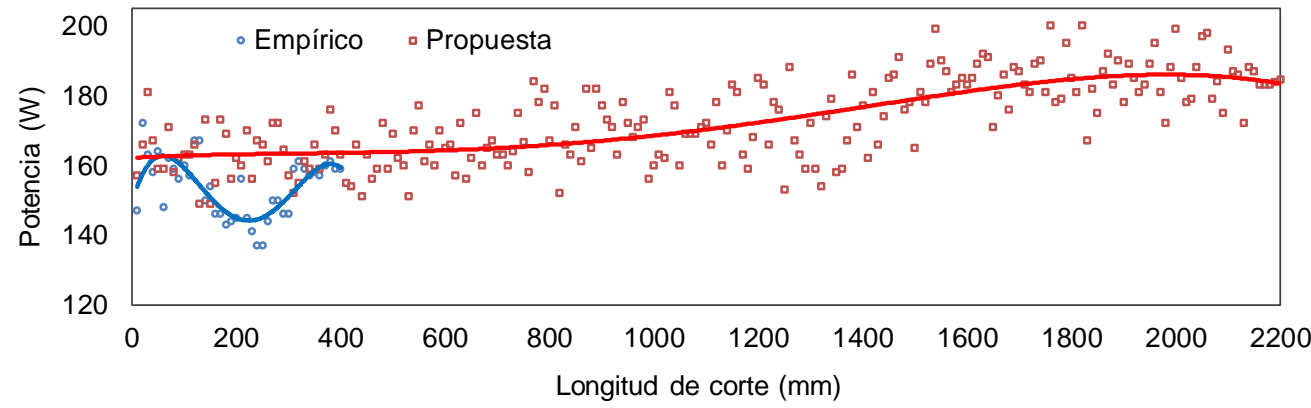

Fig. 6: Variación de potencia brocas diámetro $5 \mathrm{~mm}$.

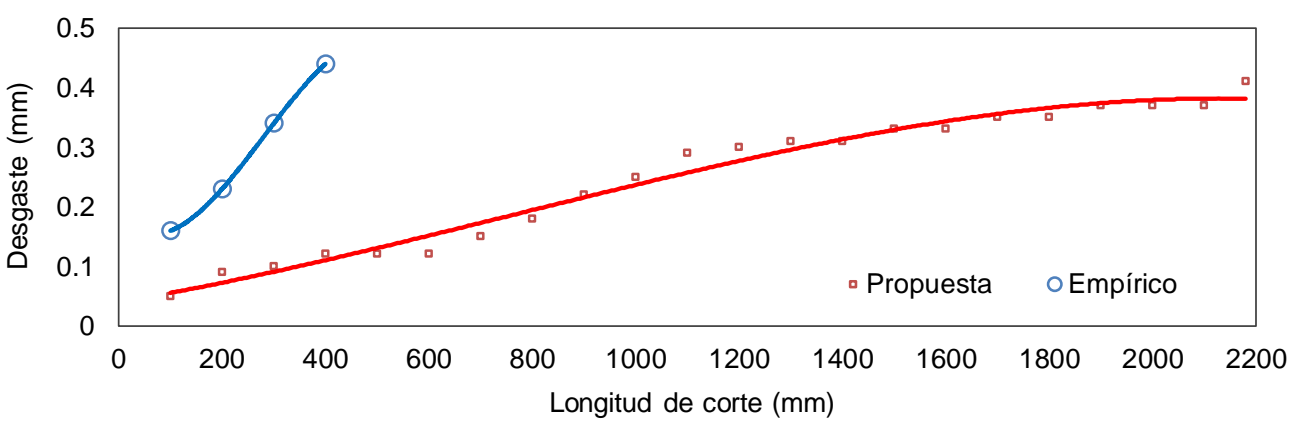

Fig. 7: Desgaste de flanco en brocas diámetro $5 \mathrm{~mm}$.
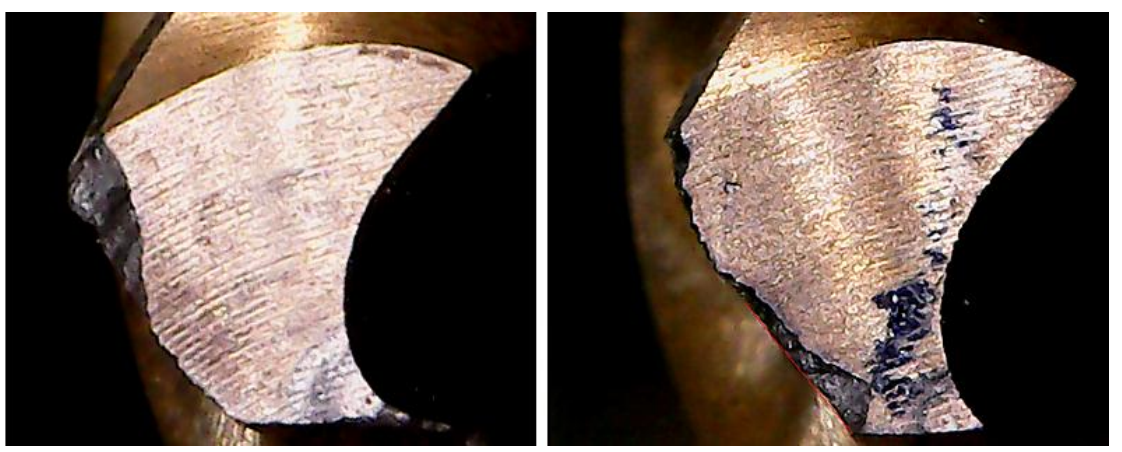

Fig. 8: Desgaste de flanco en broca $5 \mathrm{~mm}$. A la izquierda en la propuesta, a la derecha con empírico. 
En la Fig. 9 se observa el comportamiento de la potencia para la broca diámetro $8 \mathrm{~mm}$, se ha obtenido una longitud de corte de $2800 \mathrm{~mm}$ con un tiempo de corte de $27.2 \mathrm{~min}$. Se registró una potencia mínima de $315 \mathrm{~W}$ y una máxima de $728 \mathrm{~W}$. En el segundo ensayo se registra una potencia máxima de $395 \mathrm{~W}$ y mínima de $256 \mathrm{~W}$, se alcanza una longitud de $2400 \mathrm{~mm}$ y un tiempo de corte de $42.9 \mathrm{~min}$. Como se indica en la Fig. 10, el desgaste máximo con parámetros propuestos es de $0.39 \mathrm{~mm}$ mientras que con parámetros empíricos es de $0.47 \mathrm{~mm}$. Para el caso de la broca diámetro $10 \mathrm{~mm}$, se alcanzó una longitud de corte de $5250 \mathrm{~mm}$ con potencia máxima de 758 W y mínima de 499 W, en el segundo ensayo estas potencias fueron 368 W y 292 W respectivamente, se alcanzó una longitud de corte de corte de $4500 \mathrm{~mm}$ con una velocidad de avance de $57.98 \mathrm{~mm} / \mathrm{min}$. En la Fig. $11 \mathrm{se}$ puede observar la variación de la potencia. El desgaste de flanco se indica en la Fig. 12.

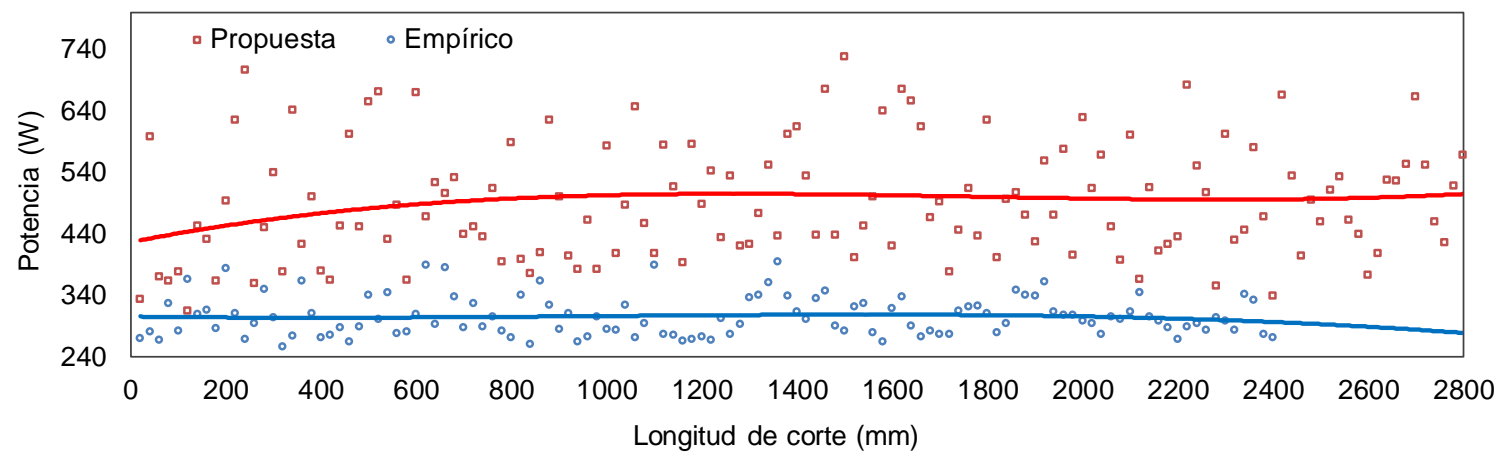

Fig. 9: Variación de potencia broca diámetro $8 \mathrm{~mm}$.

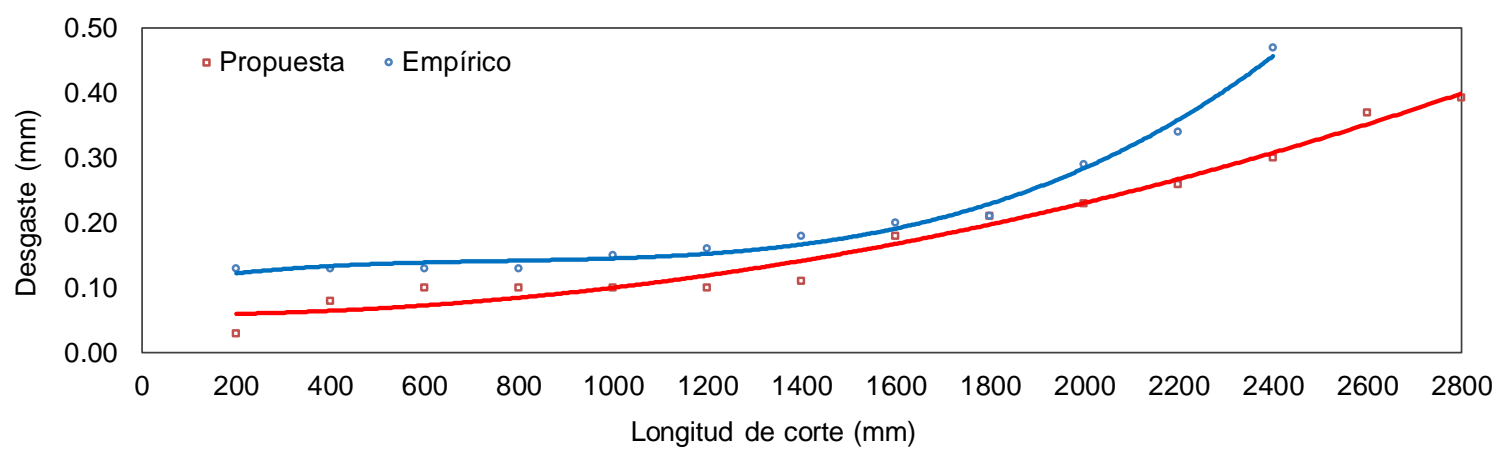

Fig. 10: Desgaste de flanco en brocas diámetro $8 \mathrm{~mm}$.

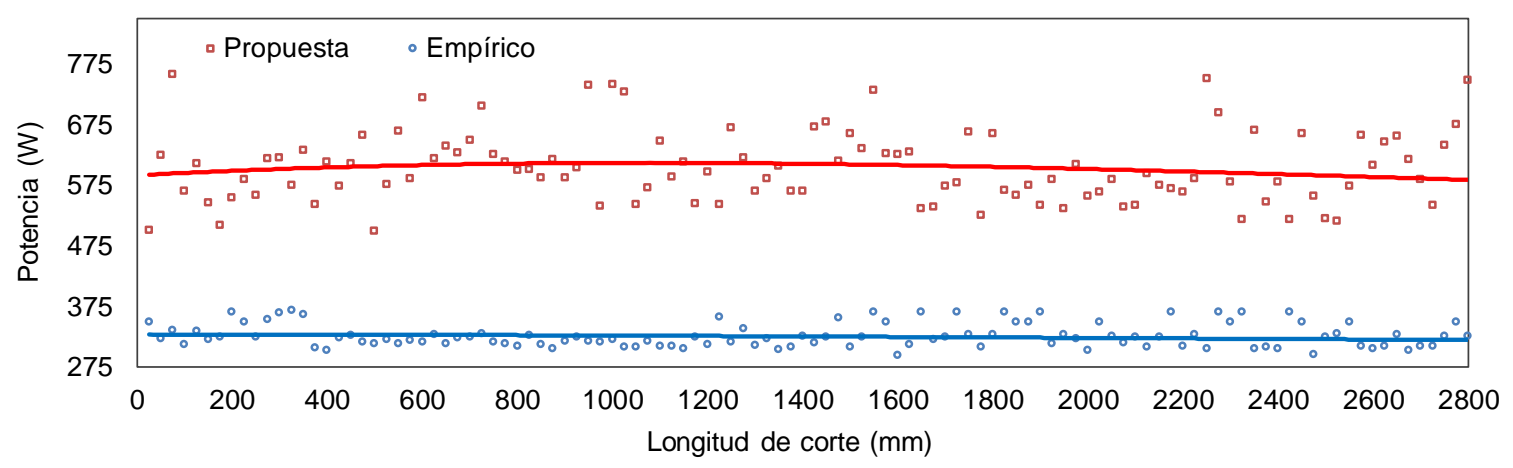

Fig. 11: Variación de potencia broca diámetro $10 \mathrm{~mm}$.

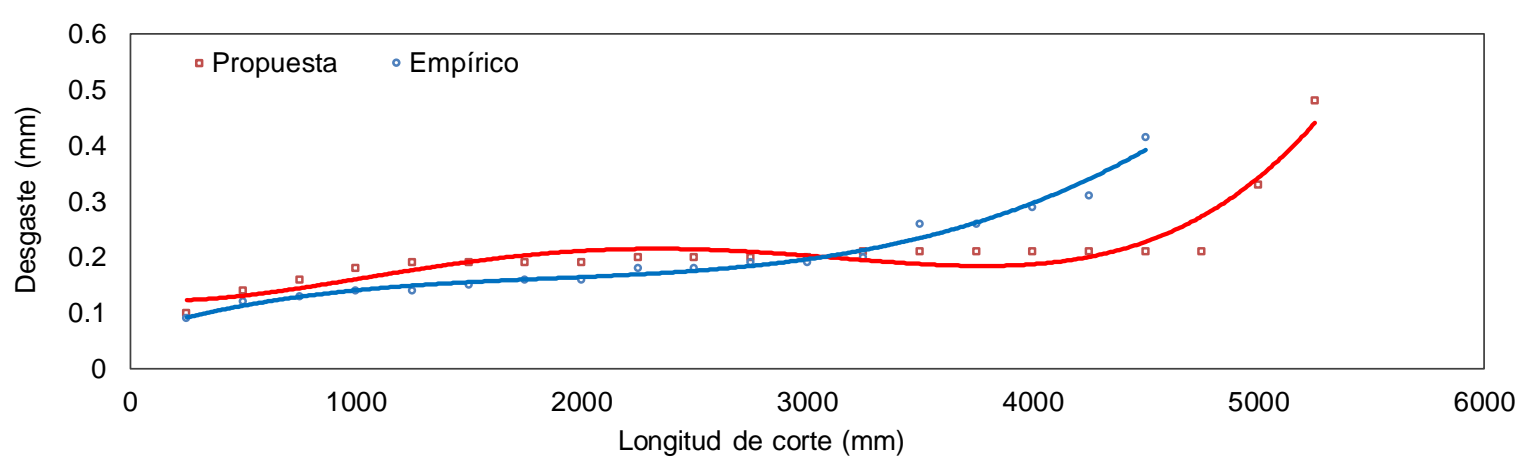

Fig.12: Desgaste de flanco en brocas diámetro $10 \mathrm{~mm}$. 
Los parámetros empíricos han demostrado tener buena eficiencia, con respecto a los resultados obtenidos en la investigación de Garg et al., (2010) en la broca diámetro $8 \mathrm{~mm}$ se obtuvo $1400 \mathrm{~mm}$ adicionales, mientras que para la broca diámetro $10 \mathrm{~mm}$ este autor reporta una duración de $135 \mathrm{~mm}$. Para las brocas diámetro 8 y $10 \mathrm{~mm}$, los parámetros propuestos alargan $14.3 \%$ más la vida útil de la herramienta en comparación con los parámetros empíricos. Al observar la Fig. 9, y la Fig. 11, se ratifica lo estipulado por Neugebauer et al., (2011) cuando menciona que; al incrementar las velocidades de corte y avance, existe un mayor consumo de potencia. Razón por la cual para el caso de las brocas 8 y $10 \mathrm{~mm}$ se debería encontrar un equilibrio entre desgaste y potencia consumida con la finalidad de optimización de energía y recursos.

\section{CONCLUSIONES}

A través de la metodología planteada se llega a las siguientes conclusiones: 1) los datos recopilados por las encuestas permitieron ratificar la hipótesis planteada sobre el uso empírico de las herramientas genéricas de taladrado por parte de las MIPYMES metalmecánicas del Ecuador; 2) la caracterización permitió llegar a concluir que las brocas ensayadas son de calidad aceptable; 3 ) los resultados obtenidos durante la experimentación demuestran que los parámetros propuestos elevan la vida útil de las brocas ensayadas y se puede observar que para la broca diámetro $5 \mathrm{~mm}$ la vida útil de la herramienta aumenta significativamente; 4) con la propuesta existe un mayor consumo de potencia; 5) la potencia fluctúa constantemente durante la operación, esto se atribuye a la falta de homogeneidad en la dureza del material de las probetas.

\section{NOTACIÓN}

Vc: Velocidad de corte $(\mathrm{m} / \mathrm{min})$

$\mathrm{N}$ : Velocidad angular (rpm)

Lc: Longitud de corte $(\mathrm{m})$

Tc: Tiempo de corte ( $\mathrm{min})$

a: avance $(\mathrm{mm} / \mathrm{rev})$

Sc: Sección de corte $\left(\mathrm{mm}^{2}\right)$

$\mathrm{D}$ : Diámetro de la herramienta $(\mathrm{mm})$

\section{AGRADECIMIENTOS}

Los autores de este artículo expresan su agradecimiento a la Escuela Superior Politécnica de Chimborazo, en especial al Ing. Rusbel Guamán, encargado de los laboratorios de la facultad de ingeniería mecánica.

\section{REFERENCIAS}

Amini, S., Alinaghian, I., y otros 3 autores, Modified drilling process of AISI 1045 steel: a hybrid optimization, https://doi.org/10.1016/j.jestch.2017.11.005, Engineering Science and Technology, and International Journal, 20(6), 16531661 (2017)

Arteaga, J. C., Cardozo, M. L., y Diniz, M. J. T., Exports to China and economic growth in Latin America, unequal effects within the región, https://doi.org/10.1016/j.inteco.2020.06.003, International Economics, 164, 1-17 (2020)

Berzosa, F., Rubio, E. M., de Agustina, B., y Davim, J. P., Geometric optimization of drills used to repair holes in magnesium aeronautical components, https://doi.org/10.3390/met10111534, Metals, 10(11), 1534 (2020)

Biermann, D., Wolf, M., y Aßmuth, R., Cutting edge preparation to enhance the performance of single lip deep hole drills, https://doi.org/10.1016/j.procir.2012.04.030, Procedia CIRP, 1, 172-177 (2012)

Cselle, T., y Barimani, A., Today's applications and future developments of coatings for drills and rotating cutting tools, https://doi.org/10.1016/0257-8972(96)80011-9, Surface and Coatings Technology, 77(2), 712-718 (1995)

Garg, S., Patra, K., y otros 3 autores, Genetically evolved radial basis function network based prediction of drill flank wear, https://doi.org/10.1016/j.engappai.2010.02.012, Engineering Applications of Artificial Intelligence, 23(7), 1112-1120 (2010)

Khan, S. A., Shamail, S., y otros 4 autores, Wear performance of surface treated drills in high speed drilling of AISI 304 stainless Steel, https://doi.org/10.1016/j.jmapro.2020.08.022, Journal of Manufacturing Processes, 58, 223-235 (2020)

Lacalle, L. N. L., Fernández, A., y otros 4 autores, Monitoring deep twist drilling for a rapid manufacturing of ligth higthstrength parts, http://doi.org/10.1016/j.ymssp.2011.02.008, Mechanical Systems and Signal Procesing, 25(7), 2745-2752 (2011)

Liu, Y., Li, H., y otros 3 autores, The impact of Chinese steel product prices based on the midstream industry chain, https://doi.org/10.1016/j.resourpol.2019.101415, Resources Policy, 63, 101415 (2019)

Lobos, J. A., y Guzmán, M. A., Estimación de la presión específica de corte en madera de pinus radiata, https://doi.org/10.4067/s0718-07642019000200223, Información Tecnológica, 30(2), 223-234 (2019)

Marques, F. M., da Silva Rocha, A., Cavaler, L. C., y Mercado, E. I., Caracterização de desgaste de brocas de hss revestidas com alcrn e não revestidas, em ensaios de furação, na usinagem de ferro fundido nodular DIN GGG 50, Revista 
Iberoamericana de Ingeniería Mecánica, 19(1), 87-104 (2015)

Neugebauer, R., Schubert, A., Reichmann, B., y Dix, M., Influence exerted by tool properties on the energy efficiency during drilling and turning operations, https://doi.org/10.1016/j.cirpj.2011.06.011, CIRP Journal of Manufacturing Science and Technology, 4(2), 161-169 (2011)

Nickel, J., Shuaib, A. N., Yilbas, B. S., y Nizam, S. M., Evaluation of the wear of plasma-nitrided and TiN-coated HSS drills using conventional and micro-PIXE techniques, https://doi.org/10.1016/S0043-1648(99)00352-X, Wear, 239(2), 155-167 (2000)

Olvera, D., Lacalle, L. N. L., y otros 4 autores, Hole making using ball helical milling on titanium alloys, https://doi.org/10.1080/10910344.2012.673958, Machining Science and Technology, 16(2), 173-188 (2012)

Patiño, C. E., y Martha de Souza, G. F., Análisis de confiabilidad para herramientas de corte aplicado al proceso de taladrado, Revista Facultad de Ingeniería Universidad de Antioquia, 36, 56-59 (2006)

Peña, B., Aramendi, G., Rivero, A., y Lacalle, L. N. L., Monitoring of drilling for burr detection using spindle torque, http://doi.org/10.1016/j.jimachtools.2005.02.006, International Journal of Machine Tools and Manufacture, 45(14), 1614-1621 (2005)

Polini, W., y Prisco, U., The estimation of the diameter error in bar turning: a comparison among three cutting force models, https://doi.org/10.1007/s00170-003-1563-8, The International Journal of Advanced Manufacturing Technology, 22(7), 465474 (2003)

Puneet, P. C., Valleti, K., y Venu Gopal, A., Influence of surface preparation on the tool life of cathodic arc PVD coated twist drills, https://doi.org/10.1016/j.jmapro.2017.05.011, Journal of Manufacturing Processes, 27, 233-240 (2017)

Quezada-Torres, W. D., Hernández-Pérez, y otros 3 autores, Evaluación integral de la transferencia de tecnologías en empresas manufactureras ecuatorianas, Ingeniería Industrial, 40(3), 295-305 (2019)

Quezada-Torres, W. D., Hernández-Pérez, G. D., y Quezada, W., Modelo de gestión tecnológica para la intensificación de la industria metalmecánica en el Ecuador: una solución conceptual, Latin American Journal of Business Management, ISSN: 2178-4833, 8(1), 222 (2017)

Ramalhete, P. S., Senos, A. M. R., y Aguiar, C., Digital tools for material selection in product design, https://doi.org/10.1016/j.matdes.2009.12.013, Materials and Design, 31(5), 2275-2287 (2010)

Tseng, M. L., Lin, S., y otros 3 autores, A causal sustainable product-service system using hierarchical structure with linguistic preferences in the Ecuadorian construction industry, https://doi.org/10.1016/j.jclepro.2019.05.140, Journal of Cleaner Production, 230, 477-487 (2019)

Urdaneta, A. J., y Borgucci, E. V., Economías de aglomeración y externalidades negativas en Ecuador, periodo 2007-2017, c, Cuadernos de Economía, 40(82), 165-191 (2021)

Vazquez, K. P. M., Giardini, C., y Ceretti, E., Cutting force modeling, https://doi.org/10.1007/978-3-642-20617-7_6399, In BT CIRP Encyclopedia of Production Engineering BT-CIRP, 315-329 (2018) 